\title{
Prediction of long-term kinetics of vaccine-elicited neutralizing antibody and time-varying vaccine-specific efficacy against the SARS-CoV-2 Delta variant by clinical endpoint
}

Xinhua Chen, MPH ${ }^{1,-}$, Wei Wang, $\mathrm{PhD}^{1,-}$, Xinghui Chen, BSc ${ }^{1}$, Qianhui Wu, MPH${ }^{1}$

Ruijia Sun, BSc ${ }^{1}$, Shijia Ge, BSc ${ }^{1}$, Nan Zheng, BSc ${ }^{1}$, Wanying $\mathrm{Lu}, \mathrm{BSc}^{1}$, Juan Yang, $\mathrm{PhD}^{1}$, Lance Rodewald, M.D. ${ }^{2}$, Hongjie Yu, $\mathrm{PhD}^{1}$

*These authors are co-first authors contributed equally to this work. Corresponding author: Hongjie Yu, Fudan University, School of Public Health, Key Laboratory of Public Health Safety, Ministry of Education, Shanghai 200032, China; E-mail: yhj@fudan.edu.cn

\section{Affiliations:}

1. School of Public Health, Fudan University, Key Laboratory of Public Health Safety, Ministry of Education, Shanghai, China

2. National Immunization Programme, Chinese Center for Disease Control and Prevention, Beijing, China

Word count (Abstract): 213

Word count (main text): 2,742 
medRxiv preprint doi: https://doi.org/10.1101/2021.09.23.21263715; this version posted September 27, 2021. The copyright holder for this preprint (which was not certified by peer review) is the author/funder, who has granted medRxiv a license to display the preprint in perpetuity.

It is made available under a CC-BY-NC-ND 4.0 International license .

Disclaimer: The views expressed are those of the authors and do not necessarily represent the institutions with which the authors are affiliated. 


\section{Abstract}

Evidence on vaccine-specific protection over time and boosting impact against the

Delta variant across different clinical endpoints and age groups is urgently needed. To address this, we used a previously published model, combined with neutralization data for four vaccines - mRNA-1273, BNT162b2, NVX-CoV2373, and CoronaVac to evaluate long-term dynamics of neutralizing antibody and to predict time-varying efficacy against the Delta variant by specific vaccine, age group, and clinical severity. We found that booster vaccination produces higher neutralization titers compared with titers observed following primary-series vaccination for all vaccines studied. We estimate the efficacies of mRNA-1273 and BNT162b2 against Delta variant infection to be $63.5 \%$ (95\%CI: 51.4-67.3\%) and 78.4\% (95\%CI: 72.2-83.5\%), respectively, 14-30 days after the second dose, and that efficacies decreased to $36.0 \%$ (95\% CI: 24.1-58.0\%) and 38.5\% (95\%CI: 28.7-49.1\%) 6-8 months later. After administration of booster doses, efficacies against the Delta variant would be $97.0 \%$ (95\%CI: 96.4-98.5\%) and 97.2\% (95.7-98.1\%). All four vaccines are predicted to provide good protection against severe illness from the Delta variant after both primary and booster vaccination. Long-term monitoring and surveillance of antibody dynamics and vaccine protection, as well as further validation of neutralizing antibody or other markers that can serve as correlates of protection against SARS-CoV-2 and its variants are needed to inform COVID-19 pandemic preparedness. 


\section{Introduction}

The ongoing coronavirus disease 2019 (COVID-19) global pandemic, caused by severe acute respiratory syndrome coronavirus 2 (SARS-CoV-2) has led to immense mortality, morbidity, and huge socioeconomic damage. ${ }^{1}$ Safe, effective, and deployable vaccines are effective tools to control virus transmission, build population-based herd immunity, and help bring the world back to pre-pandemic normalcy. Although vaccines trigger both cellular and humoral immunity, protective immunity following vaccination often decays over time, and as the virus evolves and variants emerge into prominence, protection provided by currently available COVID-19 vaccines is becoming compromised.

Although the observed dynamics of vaccine-elicited neutralizing antibody, a highly predictive bio-marker of humoral immunity, has been well-characterized, few prospective studies are available on long-term kinetics of vaccine-induced neutralizing antibody or protective efficacy/effectiveness. As of this writing, one study reported kinetics of antibodies during the three months post-vaccination with BNT162b2, ${ }^{2}$ and another showed decreased vaccine efficacy against the prototype strain during a six-month follow-up of BNT162b2 vaccination. ${ }^{3}$ For the majority of licensed COVID-19 vaccines, long-term neutralizing antibody kinetics and vaccine efficacy over time against the prototype strain and the Delta variant are not known. Additionally, the extent to which a booster dose provides protection beyond primary series vaccination is important but not known. Generating such evidence requires 
labor-intensive, time-consuming, long-term study. A recently-published modelling study predicted vaccine efficacy over time considering booster vaccination and clinical severity. ${ }^{1}$ However, the study did not evaluate vaccine-platform and age-group impact, nor did it include efficacy against infection as a modelled outcome.

Using a previously-verified model that correlated neutralizing antibodies and vaccine protection, we report predictions of long-term kinetics of vaccine-induced neutralizing antibody and time-varying vaccine efficacy against the Delta variant across different vaccines and against three clinical endpoints - infection, symptomatic COVID, and severe COVID-19. We report predicted, age-specific vaccine efficacies over time, comparing four different COVID-19 vaccines.

\section{Results}

\section{Neutralizing antibody dynamics from different vaccines}

For CoronaVac vaccine, immunogenicity data are from a phase 1/2 clinical trial in 244 healthy adults as described in the Methods section, below, and detailed in Appendix p1, Table S1. The time to reach a protective threshold was 26-31 days after the second dose of CoronaVac (Fig.1 and Appendix p7-8, Fig. S3-S4). A monotonic increase was observed until two months after the second dose of CoronaVac vaccine, with a peak neutralizing antibody titer of 52 and a seroprevalence of $76 \%$. The model estimated that individuals lost immunity 3.4 to 4.0 months after the primary two-dose series. Participant age had little effect on the kinetics of CoronaVac vaccine-induced 
SARS-CoV-2 antibody (Appendix p7, Fig. S3), and the half-life of CoronaVac vaccine-induced SARS-CoV-2 antibody was estimated to be 57 days in all participants (Appendix p8, Fig. S5). Assuming 2.4-fold reductions of antibody levels against the Delta variant compared to prototype strain, the time to the maximum titer of 23 was 2.3 months after completion of the second dose (Appendix p9, Fig. S6), a finding that was consistent between younger adults and older adults. In participants vaccinated with two primary doses plus one booster dose, the time to the maximum concentration of 187 was observed one month after the booster dose. Compared with participants vaccinated with two primary doses, a 3.8-fold change in maximum concentration was observed by addition of the booster dose (Fig. 1). The percent of participants with protective antibody titers increased to reach a peak value of $98.3 \%$ protected 28 to 30 days after the booster dose.

In our literature search, we identified three studies that reported neutralizing antibody dynamics and antibody levels after a booster dose. The studies were of BNT162b2, mRNA-1273, and NVX-CoV2373 vaccines - all two-dose primary-series vaccines with 21-28-day intervals between doses and studied with a booster dose administered 6-8 months after the second dose. Neutralizing antibodies increased after primary series vaccination and reached peaks in one month, declining to relative low levels after 6-8 months. Booster vaccination induced higher levels of neutralizing titers compared with titers induced by two primary doses. Using plaque reduction neutralizing tests, the reported neutralization titer induced by BNT162b2 for 
individuals aged 18-55 years was 497 (95\%CI: 248-979) on day 7 after the second dose, then declined to 387 (95\%CI: 245-591) one month after the second dose, and to 83 (95\% CI: 35-194) eight months after the second dose. After a booster dose, the titer increased to 1754 (95\% CI:1260 -2504) on day 7 and to 2119 (95\% CI:1257-3393) one month after the booster dose (Figure 2). The immunogenicity pattern among elderly persons (65-85 years) was similar to that of younger adults but with lower levels of neutralizing antibodies. For the mRNA-1273, neutralization titers assessed with a pseudovirus neutralization assay were 1210 (95\%CI: 840-1740) one month after the second dose, then decreased to 198 (95\%CI: 126-315) 6-8 months after the second dose, and increased to 4588 (95\%CI: 3315-6572) after a third dose (Figure 2). For NVX-CoV2373, the live-virus neutralization titer was 1581 (95\%CI: 1030-2379) two weeks after the second dose, declining to 65 (95\%CI: 41-99) six months after the second dose, and increasing to 6039 (95\%CI: 4433-7998) one month after a booster dose (Figure 2).

\section{Prediction of efficacy across different vaccines and clinical endpoints for prototype}

\section{and Delta}

Predicted efficacies were consistent with changes in neutralizing titers over time and across different clinical endpoints, and showed that predicted efficacy decreased from 14-30 days after primary series vaccination to lower values approximately 6-8 months after the second dose. Boosting increased protection from both the prototype strain and the Delta variant. For vaccine protection against infection caused by the Delta 
variant, predicted efficacies of the two mRNA-based vaccines, mRNA-1273 and BNT162b2, were 63.5\% (95\%CI: 51.4-67.3\%) and 78.4\% (95\%CI: 72.2-83.5\%) approximately 14-30 days after the second dose, respectively. Efficacies then decreased to $36.0 \%$ (95\%CI: $24.1-58.0 \%$ ) and 38.5\% (95\%CI: $28.7-49.1 \%$ ) 6-8 months after the second dose. After a booster dose, estimated efficacies against the Delta variant were $97.0 \%$ (95\%CI: 96.4-98.5\%) and 97.2\% (95.7-98.1\%). Reduction of efficacy over time against the prototype strain is less than against the Delta variant, with an approximately average protection of $91.7 \%$ (arithmetic mean) within 30 days after dose 2, 67.4\% for 6-8 months after dose 2, and nearly $99 \%$ after the booster dose for both mRNA vaccines. These predicted efficacies are higher than efficacies predicted against the prototype strain induced by vaccination with NVX-CoV2373 in a corresponding time period (Figure 3B).

For protection from symptomatic illness, the two mRNA vaccines provide good protection against both prototype and Delta strains, with over $50 \%$ protection sustained through 6-8 months after the second dose. The two mRNA vaccines can provide similar protection against the Delta variant, with $82.2 \%$ (95\%CI: 73.3-84.7\%) and $90.3 \%$ (95\%CI: 86.7-92.9\%) protection 14-30 days after the second dose, $56.1 \%$ (95\%CI: 41.5-76.5\%) and 58.1\% (95\%CI: 46.7-68.5\%) 6-8 months after the second dose, and approximately $100 \%$ after a third-dose booster. Predicted efficacy of CoronaVac against the Delta variant was lower than efficacies predicted for the mRNA vaccines after two-doses. Predicted efficacy increased to 63.8\% (95\% CI: 
44.0-80.0\%) 14-30 days after the booster dose (Figure 3B).

For severe COVID-19, predicted efficacies against the Delta variant for mRNA-1273,

BNT162b2 and CoronaVac were 98.3\% (95\%CI: 97.1-98.6\%), 98.9\% (95\%CI:

97.7-99.9\%) and 75.3\% (95\%CI: 57.9-87.3\%) between 14-30 days post dose 2; $91.6 \%$

(95\%CI: 85.8-96.6\%), 91.2\% (95\%CI: 86.8-94.3\%) and 30.1\% (95\%CI: $16.2-49.0 \%)$

6-8 months post dose 2; and 99.6\% (95\%CI: 99.5-99.8\%), 99.8\% (99.7-99.9\%), and

93.4\% (86.2-97.0\%) after the third dose (Figure 3C).

Prediction of age-specific efficacy across different vaccines and clinical endpoints

for prototype and Delta

Age-specific efficacies were estimated for BNT162b2 and CoronaVac with younger

(18-55 years) and older (65-85 years) age groups. Predicted protective efficacy was

higher for BNT162b2 than for CoronaVac over time against both prototype and Delta

variant strains and across different clinical endpoints and different age groups. The

predicted efficacies against symptomatic illness from the Delta variate for BNT162b2

were $94.7 \%$ (95\%CI: 93.1-96.0\%) 14-30 days after the second dose, $66.7 \%$ (95\%CI:

47.5-83.0\%) 6-8 months after the second dose, and 99.4\% (95\%CI: 98.7-99.7\%)

14-30 days after the booster dose. Corresponding values for Corona Vac against the

Delta variant were $34.5 \%$ (95\%CI: $18.9-54.3 \%), 8.9 \%$ (95\%CI: $4.0-18.3 \%)$ and $62.3 \%$

(95\%CI: 42.5-79.0\%). Efficacies were lower for older adults than for younger adults,

with $90.9 \%$ (95\%CI: 82.5-94.5\%) predicted protective efficacy 14-30 days after dose 
medRxiv preprint doi: https://doi.org/10.1101/2021.09.23.21263715; this version posted September 27, 2021. The copyright holder for this preprint (which was not certified by peer review) is the author/funder, who has granted medRxiv a license to display the preprint in perpetuity.

It is made available under a CC-BY-NC-ND 4.0 International license .

2, 49.5\% (95\%CI: 43.0-65.8\%) 6-8 months after the dose 2, and 99.4\% (95\%CI:

98.8-99.7\%) one month after the booster dose for BNT162b2 (Figure 4B). For the other two clinical endpoints, predicted efficacies were slightly higher for younger adults than for older adults for both prototype Delta variant strains (Figure 4A\&C). 


\section{Discussion}

Our study predicted long-term kinetics of vaccine-induced neutralizing antibodies for four COVID-19 vaccines in younger and older adults. We then predicted vaccine efficacy over time against prototype and Delta variant strains by these vaccines using three clinical endpoints in both age groups. We found similar patterns of vaccine-induced neutralizing antibodies over time, with booster vaccination producing the highest level of neutralization titers compared with primary series vaccination-induced titers. We found that predicted efficacy declined from 14-30 days after the last primary series dose to low levels 6-8 months later, and that booster doses six months after the primary series will provide greater protection than the primary series against both prototype Delta variant strains. Consistent with previous studies, we found that predicted efficacies of primary series and booster doses for the two mRNA vaccines were better than predicted efficacies from a subunit protein vaccine, NVX-CoV2373, and from an inactivated vaccine, CoronaVac. However, we predict that all vaccines can provide good protection from the Delta variant against severe illness shortly after the primary series and after booster vaccination, suggesting that timely booster vaccination of any of these prototype-based vaccines can provide protection from Delta variant illness.

Several studies have shown that vaccine-induce neutralizing antibody responses are a highly predictive proxy of vaccine protection. ${ }^{4-7}$ By correlating vaccine efficacy and level of neutralizing antibodies, protective effect over time can be estimated based on 
time-varying neutralization titers. The pattern of predicted efficacies over time are highly consistent with the kinetics of neutralizing antibody induced by vaccine administration. ${ }^{8-10}$ Doria-Rose and colleagues found that neutralizing antibodies elicited by two doses of mRNA-1273 could persisted for six months, and Barouch and colleagues reported that Ad26.COV2.S vaccine, a non-replicating adenovirus vectored vaccine, elicits humoral responses of at least eight months duration after vaccination. ${ }^{11,12}$ A real-world observational study showed that mean levels of neutralizing antibodies decline to the lower limit of sero-positivity 151-332 days after a second dose of inactivated COVID-19 vaccines. ${ }^{13}$ These results are supported by our study, in that vaccine protection decreased after primary series vaccination, and that the protection level for some vaccines 6-8 months later was less than 50\%especially for protection against variants. Our study also found that a booster dose can provide better vaccine efficacy for three clinical endpoints, a finding that is consistent with several studies showing that booster doses given six months after primary series vaccination increase neutralizing antibodies to higher levels than primary vaccination. ${ }^{14,15}$ Immunogenicity of a booster dose, boosting previously established immunity from natural infection or vaccination, and potential mechanisms of cross-protection need further study.

Compared to the dynamics of neutralizing antibodies in convalescent patients, a cohort study found that $90 \%$ of participants still had neutralization titers of $\geq 1: 20$ 6-8 months post symptom onset, and that even low levels of neutralizing antibody (1:20) 
are associated with a substantial degree of protection against COVID-19 in nonhuman primates. ${ }^{16,17}$ Although correlates of protection acquired from natural infection have not yet been established for COVID-19, and correlates of protection from illness of various severity levels remain unknown, neutralizing antibodies induced by virus infections may serve as a proxy to indicate protection. However, it should be noted that neutralizing antibodies are not the sole mechanism of protection from COVID-19; more research on the protection provided by cellular immunity is needed.

Our study showed that protective levels for different vaccines decreased by varying degrees six to eight months after primary series vaccination. Declines for a subunit protein vaccine and an inactivated vaccine were more pronounced compared with the two mRNA vaccines - especially against the Delta variant. It has been widely accepted that immunogenicity against prototype SARS-CoV-2 from mRNA vaccines is better than immunogenicity from other vaccines made with other technological platforms. This is consistent with our finding that the decline of neutralizing antibodies was smaller and slower with mRNA vaccines than for protein and inactivated vaccines. Additionally, in vitro cross neutralization assays showed that reduction of neutralizing antibodies against the Delta variant is significantly less than for vaccines made with other platforms and for convalescent sera. ${ }^{18}$ These factors likely contribute to the relatively higher and sustained predicted efficacy for mRNA vaccines. Boosting induces higher antibody responses to the prototype strain than does primary series vaccination, and the high magnitude of the response may provide 
protection against the Delta variant with different clinical severities. Maximizing neutralizing antibody response to the prototype strain, in the absence of Delta-variant-specific vaccines, may be an effective strategy to prevent or control Delta variant outbreaks.

Our study estimated vaccine-specific protection over time and the impact of boosting vaccination. Our findings provide evidence for public health decision-makers on the timing of booster doses and preparations for outbreaks. We estimated that the efficacies of BNT162b2 against the prototype strain one month to six-to-eight months after the primary series are $94.6 \%(92.5-96.8 \%)$ and $74.1 \%(69.9-78.2 \%)$, respectively. These results are similar to a real-world study that reported that efficacy peaked at $96.2 \%$ (93.3-98.1\%) within 2 months, and declined to 83.7 (95\%CI: 74.7-89.9\%) four months after the second dose, for an average decline of six percent every two months. Assuming constant rate of decline, efficacy on month six after the second dose will be $77.7 \%$, which is slightly higher than our predicted estimate of $74.1 \%$. Our study predicted that efficacy of mRNA-1273 is $90.4 \%$ (95\% CI: 87.9-93.0\%) six to eight months after the second dose, which is comparable to the reported efficacy $(92.4 \%$, 95\%CI: 84.3-96.8\%). Our study predicted age-specific vaccine protection by different time periods after two-dose primary series, showing that efficacy for younger adults is slightly higher than for older adults due to differences in immunogenicity by age group. ${ }^{19,20}$ A previous study estimated variant-specific efficacy against symptomatic and severe infection, and also modeled boosting impact after primary series 
vaccination. ${ }^{1}$ This study did not estimate efficacy by different vaccines and vaccine protection against SARS-CoV-2 infection between different time periods after primary series vaccination. Furthermore, the influence of age was not considered in the study, thus, age-specific efficacies estimates from different vaccines were not made. In contrast, our current study provides a timely prediction of vaccine efficacy against both prototype strain and the Delta variant across different vaccines, clinical endpoints, and time since primary series vaccination, and also estimates impact of booster doses.

Our study has several limitations. First, our predictions depend on level of neutralizing antibodies, without taking other immunologic mechanisms of humoral and cellular immunity into account. However, previous studies showed that neutralizing antibodies are highly predictive of immune protection. Second, to enhance comparability between different studies with different neutralization assays, we normalized the neutralization titers in each study by the mean titers in convalescent sera reported in Phase I/II trials against prototype strains that used the same type of neutralization assay. However, other sources of heterogeneity, such as lab-to-lab variation and experimental procedures could not be accounted for. Third, the n-fold-reduction of neutralization titers of the Delta variant was not available for certain time periods for mRNA-1273. Instead, we used the n-fold-change from the Beta variant - a variant with more significant immune-escape capacity - to provide a conservative estimated for our analyses. We also conducted a sensitivity analysis by 
using n-fold-reduction from the Gamma variant (a variant with less significant immune-escape capacity than Delta).

In conclusion, our study predicted time-varying vaccine protection from prototype SARS-CoV-2 and the Delta variant and the increase of protection that results from booster dose administration. We estimated protection by four COVID-19 vaccines from three clinical endpoints in two age groups. Our findings suggest that, regardless of age group, timely boosting with SARS-CoV-2-prototype-based vaccines can provide protection against the Delta variant, although with better performance from mRNA vaccines than from protein and inactivated vaccines. Irrespective of vaccine technology, however, booster doses will effectively prevent symptomatic and severe COVID-19 caused by the Delta variant. Long-term monitoring and surveillance of antibody dynamics and vaccine protection, as well as further validation of neutralizing antibody or other markers that can serve as correlates of protection are urgently needed to inform COVID-19 pandemic preparedness. 


\section{References}

1. Cromer, D., et al. SARS-CoV-2 variants: levels of neutralisation required for protective immunity. medRxiv, 2021.2008.2011.21261876 (2021).

2. Favresse, J., et al. Antibody titres decline 3-month post-vaccination with BNT162b2. Emerg Microbes Infect 10, 1495-1498 (2021).

3. Thomas, S.J., et al. Six Month Safety and Efficacy of the BNT162b2 mRNA COVID-19 Vaccine. 2021.2007.2028.21261159 (2021).

4. Khoury, D.S., et al. Neutralizing antibody levels are highly predictive of immune protection from symptomatic SARS-CoV-2 infection. Nature medicine (2021).

5. Feng, S., et al. Correlates of protection against symptomatic and asymptomatic SARS-CoV-2 infection. medRxiv, 2021.2006.2021.21258528 (2021).

6. Earle, K.A., et al. Evidence for antibody as a protective correlate for COVID-19 vaccines. Vaccine 39, 4423-4428 (2021).

7. Koch, T., Mellinghoff, S.C., Shamsrizi, P., Addo, M.M. \& Dahlke, C. Correlates of Vaccine-Induced Protection against SARS-CoV-2. Vaccines 9(2021).

8. Pfizer, I. Second Quarter 2021 Earnings Teleconference. Vol. 2021 (2021).

9. NOVAVAX, I. Second Quarter 2021 Financial Results and Operational Highlights. Vol. 2021 (2021).

10. Moderna, I. Moderna 2Q 2021 Earnings Call. Vol. 2021 (2021).

11. Doria-Rose, N., et al. Antibody Persistence through 6 Months after the Second 
Dose of mRNA-1273 Vaccine for Covid-19. (2021).

12. Barouch, D.H., et al. Durable Humoral and Cellular Immune Responses 8

Months after Ad26.COV2.S Vaccination. N Engl J Med (2021).

13. Zhang, H., et al. Studies on the level of neutralizing antibodies produced by inactivated COVID-19 vaccines in the real world. 2021.2008.2018.21262214 (2021).

14. Pan, H., et al. Immunogenicity and safety of a third dose, and immune persistence of CoronaVac vaccine in healthy adults aged 18-59 years: interim results from a double-blind, randomized, placebo-controlled phase 2 clinical trial. 2021.2007.2023.21261026 (2021).

15. Sadoff, J., et al. Durability of antibody responses elicited by a single dose of Ad26.COV2.S and substantial increase following late boosting. 2021.2008.2025.21262569 (2021).

16. Gao, Q., et al. Development of an inactivated vaccine candidate for SARS-CoV-2. Science 369, 77-81 (2020).

17. Yu, J., et al. DNA vaccine protection against SARS-CoV-2 in rhesus macaques. Science 369, 806-811 (2020).

18. Chen, X., et al. Neutralizing antibodies against SARS-CoV-2 variants induced by natural infection or vaccination: a systematic review and pooled meta-analysis. Clinical Infectious Diseases (2021).

19. Kontopoulou, K., et al. Immunogenicity of the BNT162b2 mRNA COVID-19 Vaccine in Elderly People Over 85 Years of Age in Greece. (SSRN, 2021). 
medRxiv preprint doi: https://doi.org/10.1101/2021.09.23.21263715; this version posted September 27, 2021. The copyright holder for this preprint (which was not certified by peer review) is the author/funder, who has granted medRxiv a license to display the preprint in perpetuity.

It is made available under a CC-BY-NC-ND 4.0 International license .

20. Frenck, R.W., et al. Safety, Immunogenicity, and Efficacy of the BNT162b2

Covid-19 Vaccine in Adolescents. New England Journal of Medicine (2021). 


\section{Figure legend}

Figure 1. CoronaVac vaccine-induced SARS-CoV-2 neutralizing antibody response and corresponding seroprevalences after two primary doses plus one booster dose of Corona Vac vaccine.

(A) Fitted SARS-CoV-2 antibody titer dynamics; (B) Fitted seroprevalence (with a protective threshold of 33 for seropositivity).

Figure 2. Vaccine-induced SARS-CoV-2 neutralizing antibody response by time periods and natural-infection-induced neutralizing antibody response for convalescent patients in clinical trials.

A) mRNA-1273, B) BNT162b2, C) NVX-CoV2373, and D) CoronaVac. The number on the top of the bar represents the GMT and the vertical line represents the $95 \%$ confidence interval. The neutralization assay for mRNA-1273 is a pseudovirus neutralization assay, while virus neutralization assays were used for the other vaccines.

Figure 3. Predicted time-varying efficacy against both prototype strains and the Delta variant across three clinical endpoints. A) SARS-CoV-2 infection, B) Symptomatic COVID-19, and C) Severe COVID-19. The number on the top of the bar represents the GMT and the vertical line represents the $95 \%$ confidence interval.

Figure 4. Predicted age-specific time-varying efficacy against both prototype 
medRxiv preprint doi: https://doi.org/10.1101/2021.09.23.21263715; this version posted September 27, 2021. The copyright holder for this preprint (which was not certified by peer review) is the author/funder, who has granted medRxiv a license to display the preprint in perpetuity. It is made available under a CC-BY-NC-ND 4.0 International license .

strains and the Delta variant across three clinical endpoints. A) SARS-CoV-2 infection, B) Symptomatic COVID-19, and C) Severe COVID-19. The number on the top of the bar represents the GMT and the vertical line represents the $95 \%$ confidence interval. 


\section{Methods}

\section{Data sources}

\section{Individual-level neutralizing antibody titer data for CoronaVac}

Randomized, double-blind, placebo-controlled, phase 1/2 clinical trials of CoronaVac in healthy adults aged 18 years and older were performed in Jiangsu province and Hebei province, China, as previously detailed. ${ }^{14,21}$ In those studies, participants aged 18 years or older and vaccinated with CoronaVac were eligible for a study of the antibody kinetics of CoronaVac vaccine-induced SARS-CoV-2 neutralizing antibody. Subjects were given $3 \mu \mathrm{g}$ doses in $0.5 \mathrm{~mL}$ of aluminum hydroxide diluent of CoronaVac vaccine intramuscularly on days 0 and 28 . To evaluate persistence of CoronaVac vaccine-induced neutralizing antibody titers, blood specimens were obtained on: days 0,28 , and 56 post the first dose; 6 months after two-doses; and days 7 and 14 or 28 after a third dose (Appendix p2, Fig. S1). Neutralizing antibody titers to infective SARS-CoV-2 (virus strain SARS-CoV-2/human/CHN/CN1/2020, GenBank number MT407649.1) were quantified using a micro cytopathogenic effect assay, previously described. ${ }^{22}$

\section{Neutralizing antibody dynamics with different vaccines with time-specific}

aggregated data collected from a comprehensive literature search

Using predefined search terms, we conducted a search for studies that reported dynamics of neutralizing antibodies and impact of a booster dose among vaccine recipients in five databases - three peer-reviewed databases (PubMed, Embase, and 
Web of Science) and two preprint servers (medRxiv, bioRxiv) (Appendix p10, Table

S4). We obtained official reports from vaccine companies to supplement retrieved data. From the eligible studies, we abstracted vaccine name, vaccine developer, numbers and ages of study participants, vaccination schedule, type of neutralization assay (live or pseudo virus), and geometric mean titers (GMTs) over time after vaccination (Appendix p12, Table S5). For studies that did not report 95\% confidence intervals, we digitized individual titers from figures using a digital extraction tool and calculated the intervals. We only extracted titers expressed as reciprocal dilution of serum that neutralizes or inhibits $50 \%$ of the virus (e.g., NT50, PRNT50, etc.). For titers lower than the limit of detection, we assumed the titer to be half (e.g., we assumed a titer of 10 when " $<20$ " was reported).

\section{In vitro cross neutralization titer against prototype and Delta variant strains}

We conducted a systematic search to update a previously-reported meta-analysis of in-vitro neutralization titers in individuals who have been vaccinated with prototype-strain-based vaccines against both SARS-CoV-2 prototype strains and variants. ${ }^{18} \mathrm{~N}$-fold-reduction of neutralizing antibodies against the Delta variant compared to the prototype strain were calculated for different types of neutralization assays (Appendix p15, Table S6). For CoronaVac vaccine, which has no reported n-fold-reduction against the Delta variant, we used n-fold-reduction data from a study of an inactivated vaccine developed and manufactured in India (BBV152) that used the same neutralization assay as used for CoronaVac. In official reports for the two 
mRNA vaccines, BNT162b2 and mRNA-1273, neutralization assays for prototype and Delta strains varied by sampling period used. We extracted mean n-fold-changes of neutralization titer against the Delta variant (compared to the prototype strain) and corresponding $95 \%$ confidence intervals.

\section{Statistical analysis}

Neutralizing antibody dynamics fitted by GAM model with individual data for

\section{CoronaVac}

Titers of CoronaVac vaccine-induced SARS-CoV-2 neutralizing antibody were log-transformed before statistical analyses. Different from our previously qualitative analyses of CoronaVac vaccine-induced antibody titer data, ${ }^{14,21}$ kinetics of SARS-CoV-2 neutralizing antibody after vaccination were fitted using a generalized additive model (GAM) that allows for flexible specification of dependence of response on covariate. Computational methods were implemented with the CRAN R-package repository 'mgcv'. For modelling dynamics of SARS-CoV-2 neutralizing antibody, we assumed a GAM model with Gaussian distribution, which is commonly used to model antibody titers that involve evaluation of vaccine efficacy. Comparisons between models were made based on Bayesian information criteria (BIC) and Akaike information criteria (AIC). A binomial logistic generalized additive model was used to describe dynamic patterns of seroprevalence of vaccine-induced SARS-CoV-2 neutralizing antibody following vaccination. As used by Khoury DS et al, ${ }^{4}$ a protective threshold of 33 was selected. Given curve-fitting uncertainties of the 
antibody titers kinetics in the 120-209 days after the first-dose of CoronaVac due to a paucity of observed immunogenicity data, we used multivariate imputation methods to impute immunogenicity data using immunogenicity data from participants who received other dosing schedules. Details of GAM smoothing and multivariate imputations are in the Appendix.

\section{Predicting vaccine efficacy over time across different vaccines and clinical}

\section{endpoints}

We used the model to predict vaccine protection against prototype strain based on the relationship between neutralizing antibody level and vaccine efficacy (equation 1); we used an integral based on normal distribution of neutralization level to calculate the probability of being protected (equation 2).

$$
\begin{gathered}
E_{I}\left(n \mid n_{50}, k\right)=\frac{1}{1+e^{-k\left(n-n_{50}\right)}},(1) \\
P\left(n_{50}, k, \mu_{\mathrm{s}}, \sigma_{\mathrm{s}}\right)=\int_{-\infty}^{+\infty} \mathrm{E}_{I}\left(n \mid n_{50}, k\right) f\left(n \mid \mu_{\mathrm{s}}, \sigma_{\mathrm{s}}\right) d n,(2)
\end{gathered}
$$

A logistic model was used to model the relationship for equation (1), where $E_{I}$ is the vaccine efficacy given the log-transformed neutralizing antibody titer $\mathrm{n}$, and $n_{50}$ is the neutralization titer at which an individual will have a 50\% protective efficacy. The parameter $k$ controls the steepness of the logistic function. The logistic relationship for different clinical endpoints was constructed by changing the $n_{50}$ and $k{ }^{4}$ For equation (2), assuming that neutralizing antibodies follow a normal distribution with mean $\mu_{s}$ and standard deviation $\sigma_{s}, f$ indicates the probability density function of neutralization titer, and $P$ represents the proportion of vaccinated population for a 
study $s$ that will be protected. To enhance comparability between different studies with different neutralization assays, the neutralization titer $\left(\mu_{s}\right)$ was normalized in each study with the means of titers in corresponding convalescent individuals reported in Phase I/II trials against prototype strains. Confidence intervals of predicted efficacy against the prototype strain were estimated by using the Hessian $\mathrm{H}$ and standard error $($ s.e. $=\operatorname{sqrt}(\operatorname{diag}(\mathrm{H}-1))) ; 95 \% \mathrm{CIs}$ were calculated as $\pm 1.96 *$ s.e. of the estimated parameters.

For predictions of vaccine efficacy against the Delta variant, we added log-transformed n-fold-reduction of the Delta variant on the neutralizing antibody level into equation (3), and predicted variant-specific efficacy by using equations (1) and (2).

$$
\mu_{s}^{v}=\mu_{s}+\bar{F}^{v},(3)
$$

Where $F^{v}$ is the mean log-transformed n-fold-change (vaccine-specific) in neutralization titer against the Delta variant, $\mu_{s}$ is the normalized neutralization titer (vaccine-specific) against the prototype strain, and $\mu_{s}^{v}$ is the normalized neutralization titer (vaccine-specific) for the Delta variant. Confidence intervals of predicted efficacy against the Delta variant were calculated by imputing the $95 \%$ confidence intervals of the $\mathrm{n}$-fold changes of neutralization titers.

\section{References}

21. Li, M., et al. A booster dose is immunogenic and will be needed for older 
adults who have completed two doses vaccination with CoronaVac: a randomised, double-blind, placebo-controlled, phase 1/2 clinical trial. 2021.2008.2003.21261544 (2021).

22. Wu, Z., et al. Safety, tolerability, and immunogenicity of an inactivated SARS-CoV-2 vaccine (CoronaVac) in healthy adults aged 60 years and older: a randomised, double-blind, placebo-controlled, phase 1/2 clinical trial. Lancet Infect Dis (2021). 


\section{Role of the funding source}

The funder had no role in study design, data collection, data analysis, data interpretation, or writing of the report. The corresponding author had full access to all the data in the study and had final responsibility for the decision to submit for publication.

\section{Ethics statement}

All data used in this paper were in the public domain. Ethics committees of School of public health, Fudan university states that ethnical approval of re-use of these secondary data is not required.

\section{Contributors}

H.Y. designed and supervised the study. X.C., W.W. did the literature search, set up the database and did all statistical analyses. X.C. and W.W. co-drafted the first version of the article. Xing.C., Q.W., R.S., W.L., N.Z., and S.G. helped with checking data and did the figures. L.R, J.Y, and H.Y. commented on the data and its interpretation, revised the content critically. All authors contributed to review and revision and approved the final manuscript as submitted and agree to be accountable for all aspects of the work.

\section{Declaration of interests}

H.Y. has received research funding from Sanofi Pasteur, and Shanghai Roche 
medRxiv preprint doi: https://doi.org/10.1101/2021.09.23.21263715; this version posted September 27, 2021. The copyright holder for this preprint (which was not certified by peer review) is the author/funder, who has granted medRxiv a license to display the preprint in perpetuity. It is made available under a CC-BY-NC-ND 4.0 International license .

Pharmaceutical Company; None of those research funding is related to COVID-19.

All other authors report no competing interests.

\section{Acknowledgments}

This study was funded by the Key Program of the National Natural Science

Foundation of China (82130093). 


\section{A. mRNA-1273}

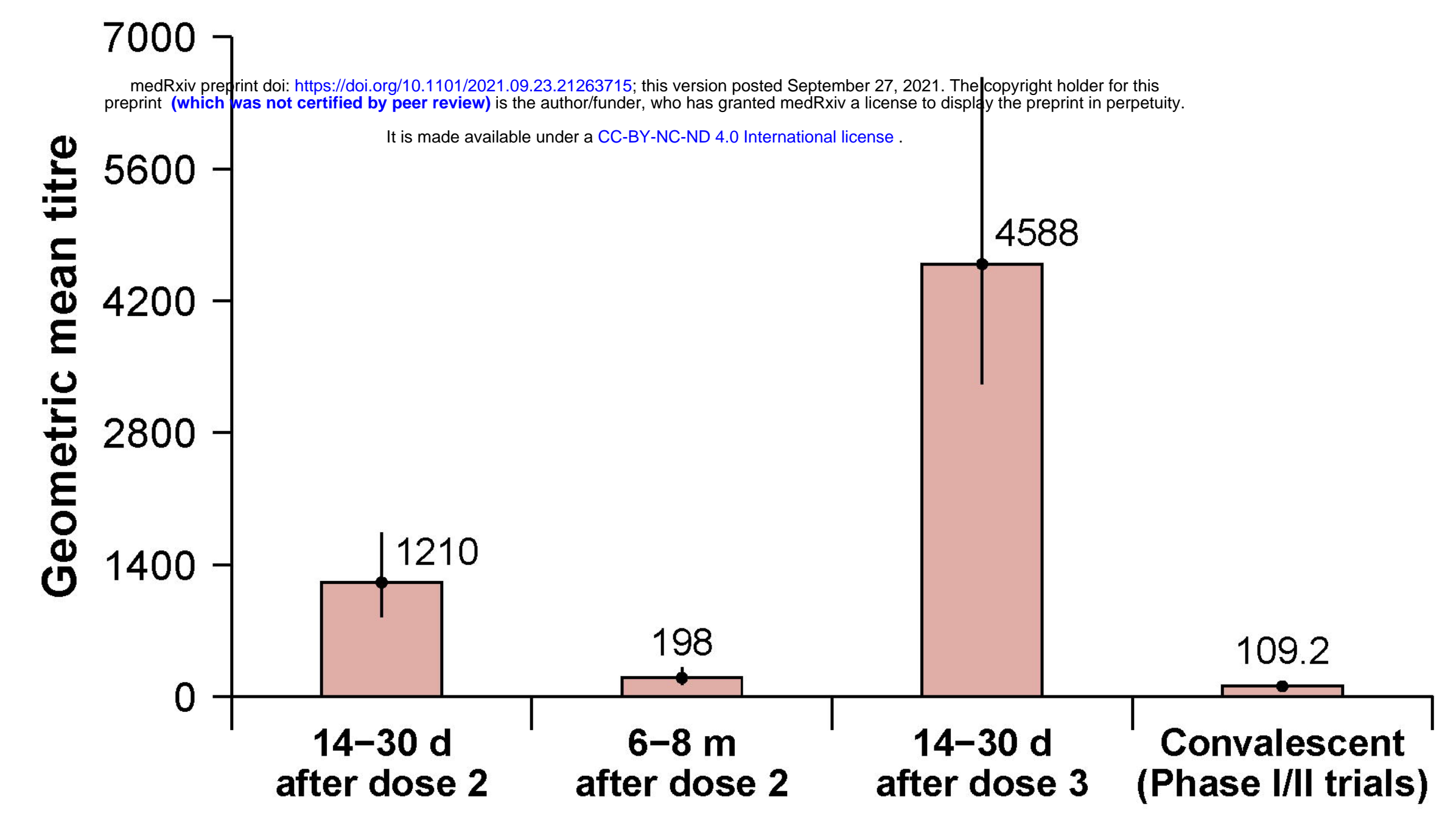

\section{NVX-CoV2373}

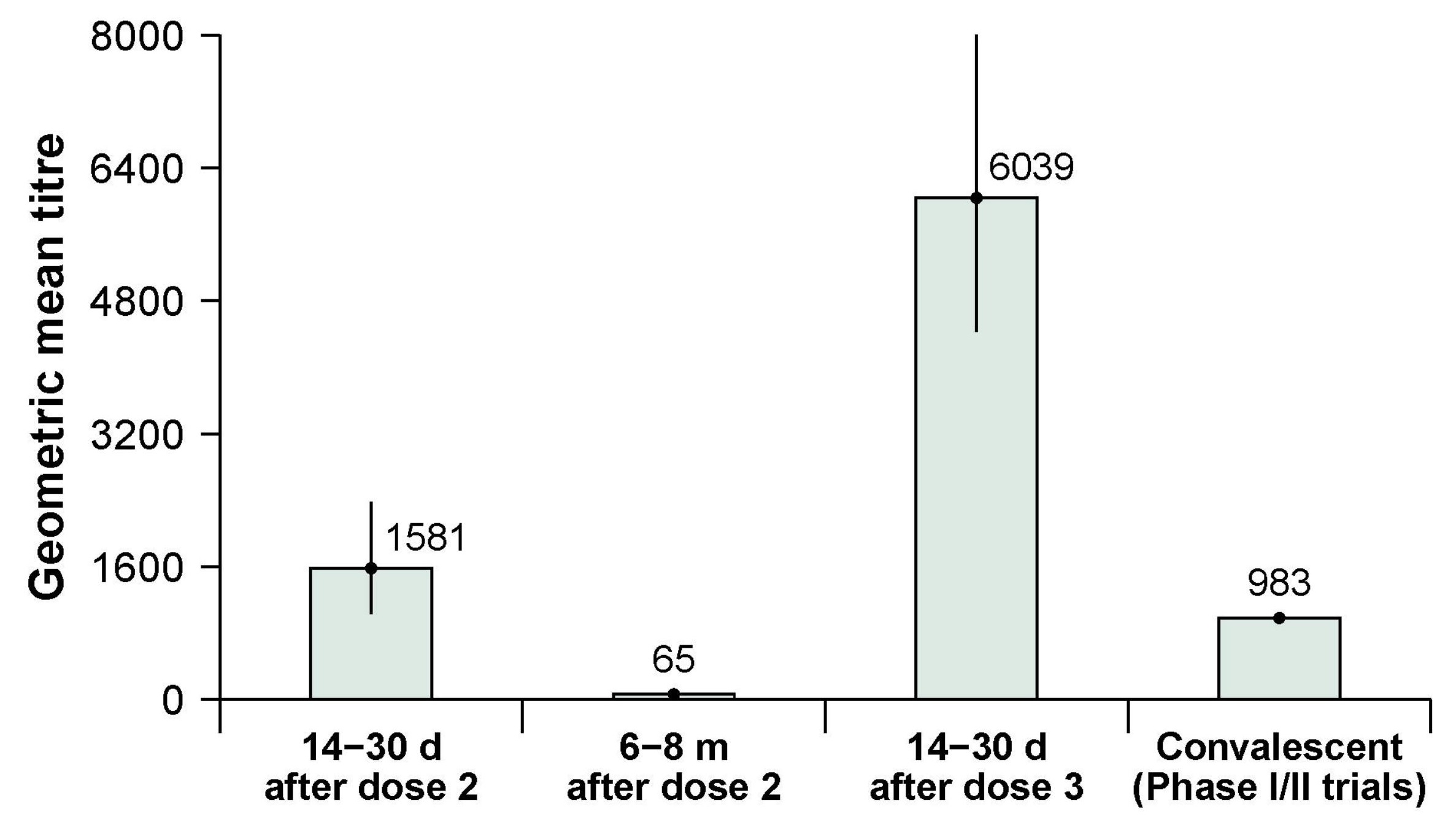

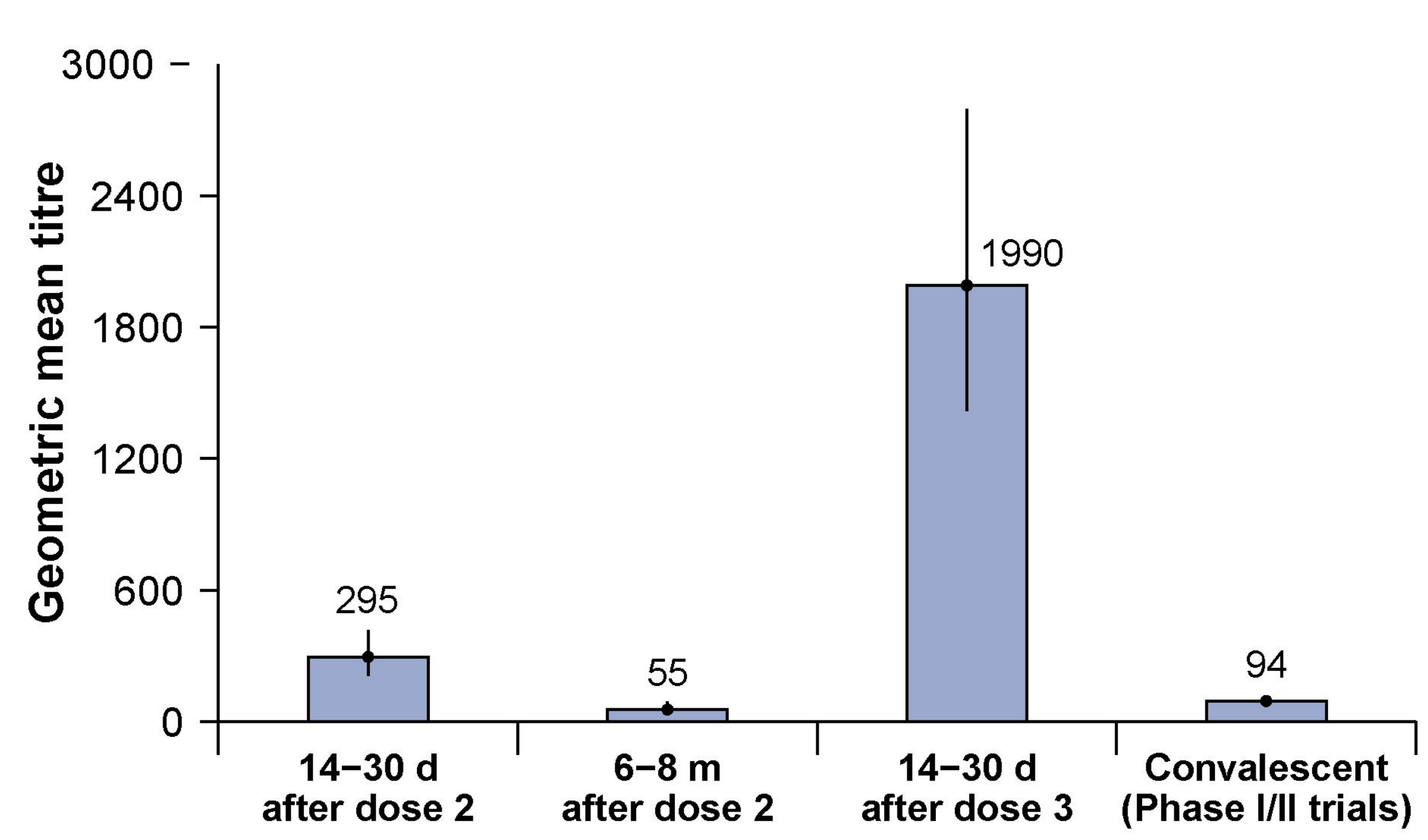

\section{CoronaVac}

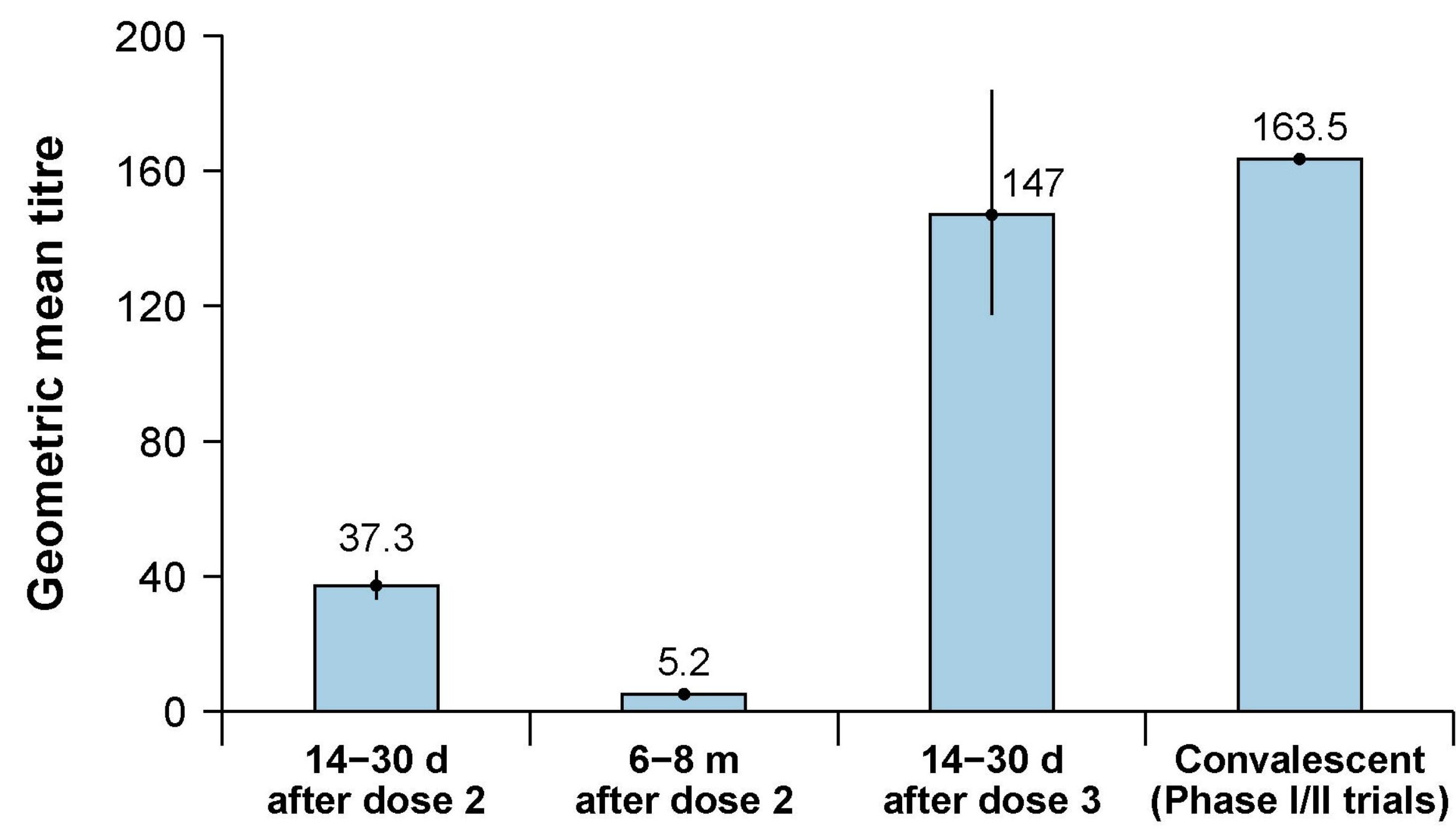




\section{A. SARS-CoV-2 infection}

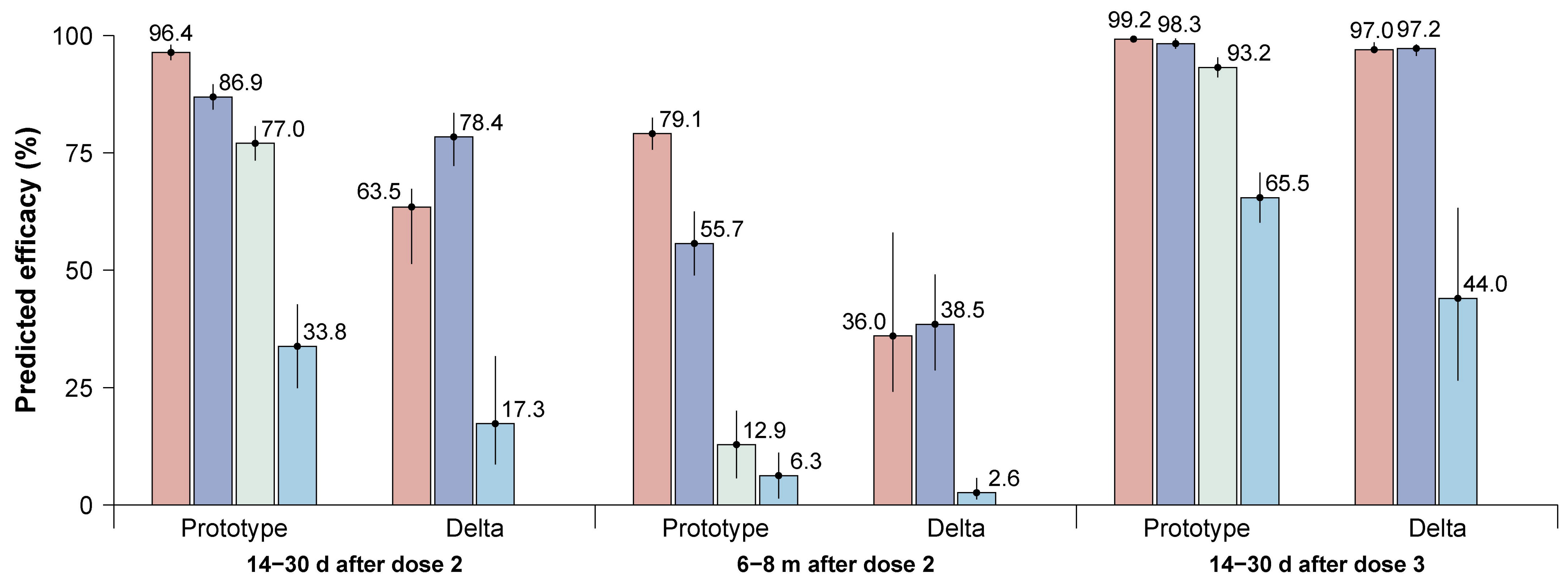

\section{B. Symptomatic COVID-19}

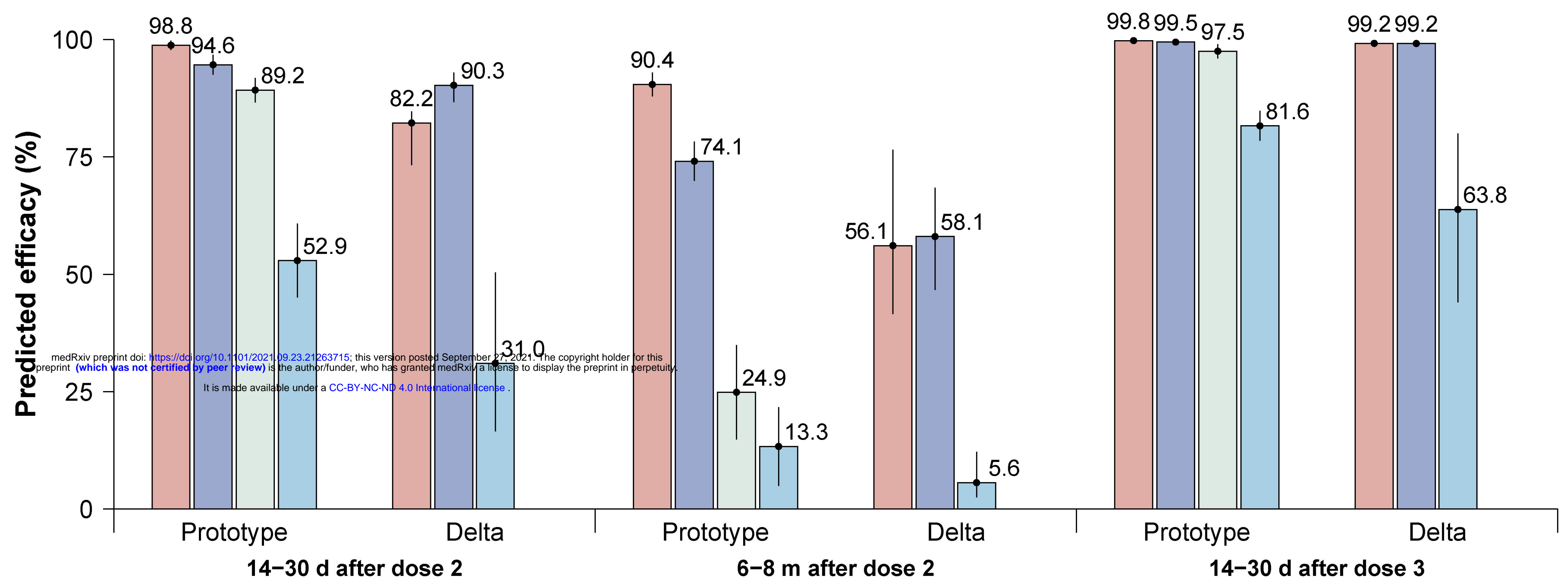

\section{Severe COVID-19}

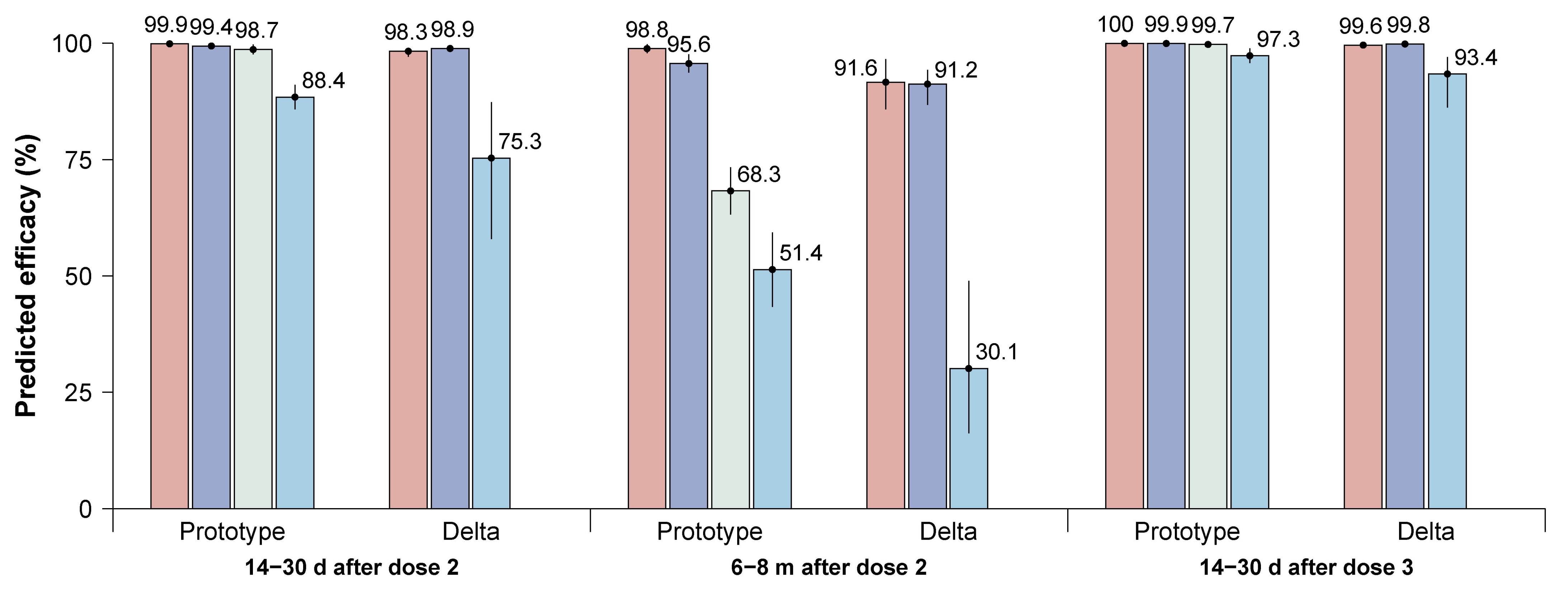


BNT162b2

18-55 yrs

65-85 yrs

CoronaVac

A. SARS-CoV-2 infection

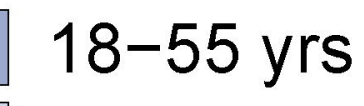

65-85 yrs

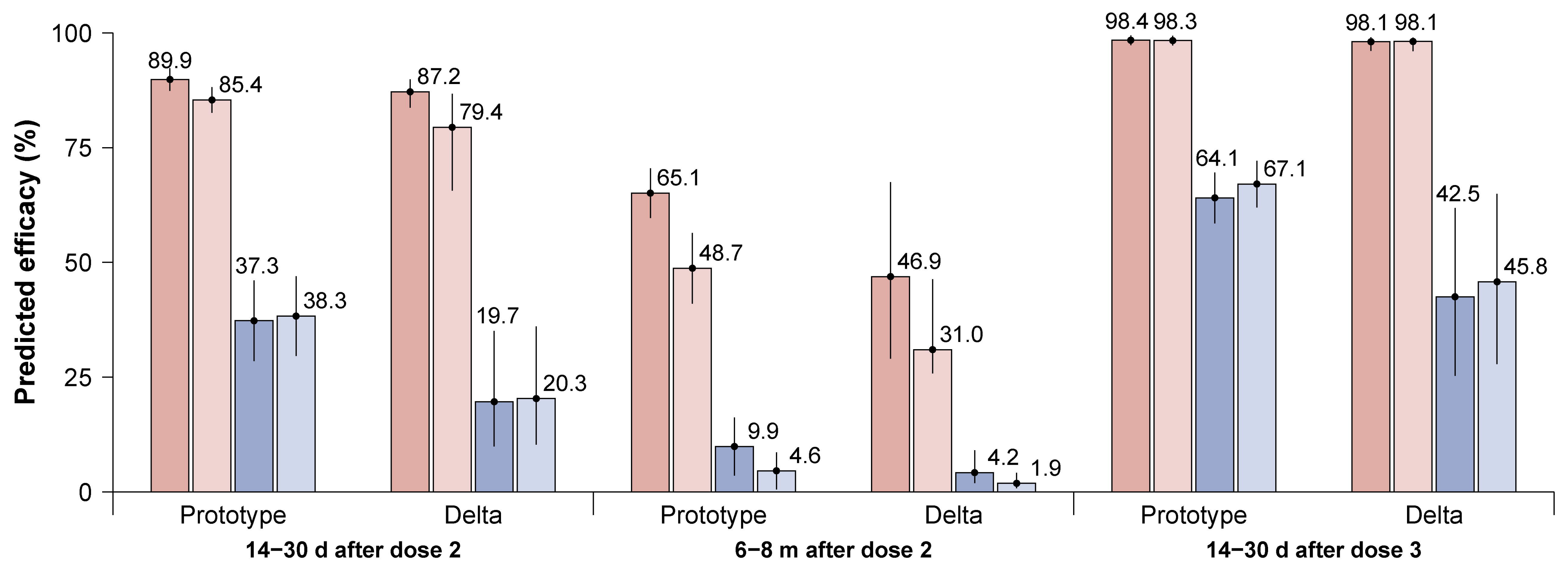

B. Symptomatic COVID-19

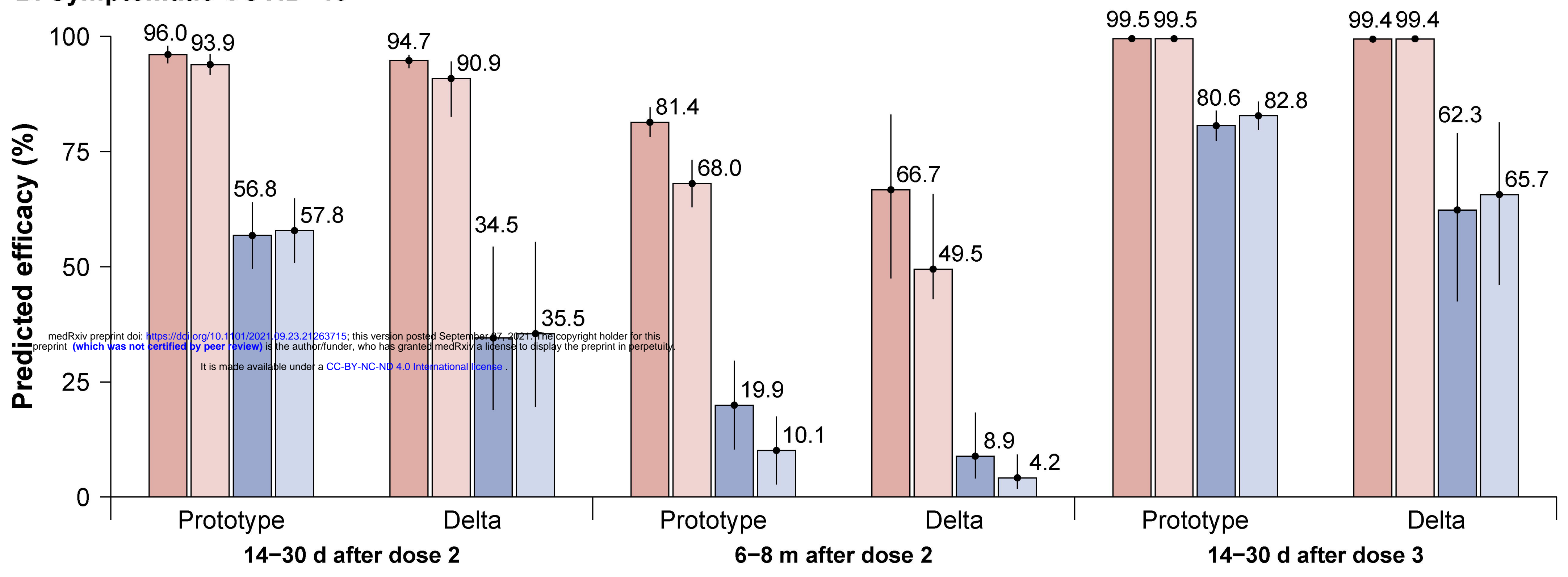

C. Severe COVID-19

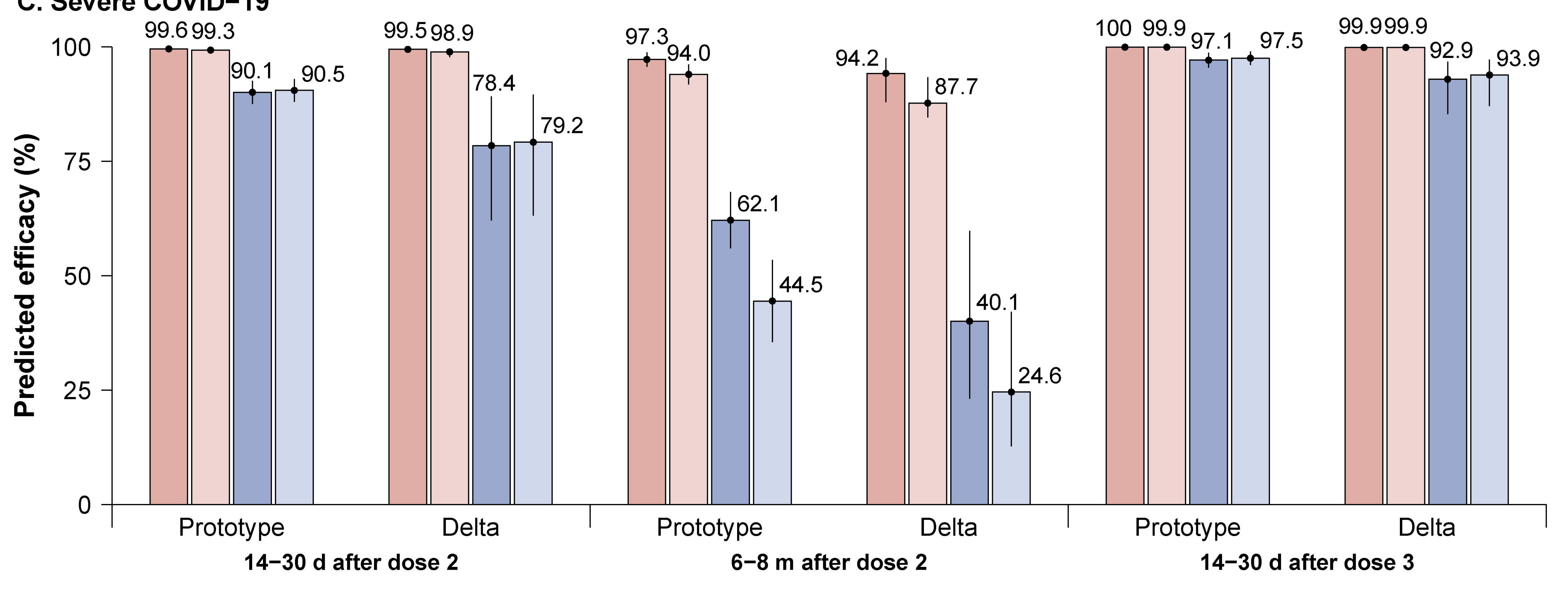

